Supporting Information

\title{
Multi-objective optimization of graded, hybrid micropillar wicks for capillary-fed evaporation
}

Tanya Liu ${ }^{l}$, Mehdi Asheghi ${ }^{l}$, Kenneth E. Goodson ${ }^{1, *}$

${ }^{1}$ Department of Mechanical Engineering, Stanford University, Stanford, CA 94305, United States 


\section{SECTION I: THERMAL RESISTANCE NETWORK VALIDATION}

In order to validate the assumptions made for our thermal resistance network model, we compared our results against the full numerical simulation results of Vaarstra et al. [1] for the effective heat transfer coefficient due to evaporation from uniform micropillar arrays. The numerical approach by Vaarstra et al. uses a finite element simulation to calculate the evaporator temperature and evaporative heat flux from the meniscus surface through a solution to the linearized Boltzmann Transport Equation. The results are presented in terms of a local effective heat transfer coefficient $h_{e}$, where

$$
h_{e}=\frac{q^{\prime \prime}}{T_{e}-T_{s a t}}
$$

and $T_{e}$ and $T_{\text {sat }}$ represent the temperatures at the micropillar base and far-field vapor, respectively. Similar to our model, their approach captures the variation in meniscus curvature along the length of the wick and results in an effective heat transfer coefficient that varies as a function of $x$.

Our results can be directly compared against theirs by converting the calculated thermal resistances for each unit cell into a local effective heat transfer coefficient as

$$
h_{e}=\left(R_{i} A_{i}\right)^{-1}
$$

where $R_{i}$ represents the resistance of an individual unit cell as described in Eqn. 6 of the manuscript, and $A_{i}$ represents the unit cell area. As seen in Figure S1, the resistance network model shows good agreement with the full numerical simulation results of Vaarstra et al. for the effective heat transfer coefficient along the wick at position $x / L$ relative to the liquid inlet location. Results are shown for the case of a uniformly heated micropillar array with $d=10 \mu \mathrm{m}, p=30 \mu \mathrm{m}$, and $h=25 \mu \mathrm{m}, q$ " $=$ $82 \mathrm{~W} / \mathrm{cm}^{2}$, and length $L=5 \mathrm{~mm}$. The results also show excellent agreement in the prediction of average heat transfer coefficient, where $h_{e, a v g}=76.7 \mathrm{kWm}^{-2} \mathrm{~K}^{-1}$ and $75.7 \mathrm{kWm}^{-2} \mathrm{~K}^{-1}$ for our resistance network model versus the full simulation model, respectively. This confirms that the assumptions made in our simplified thermal resistance network model can adequately predict the heat transfer in the micropillar array geometries considered in this study. 


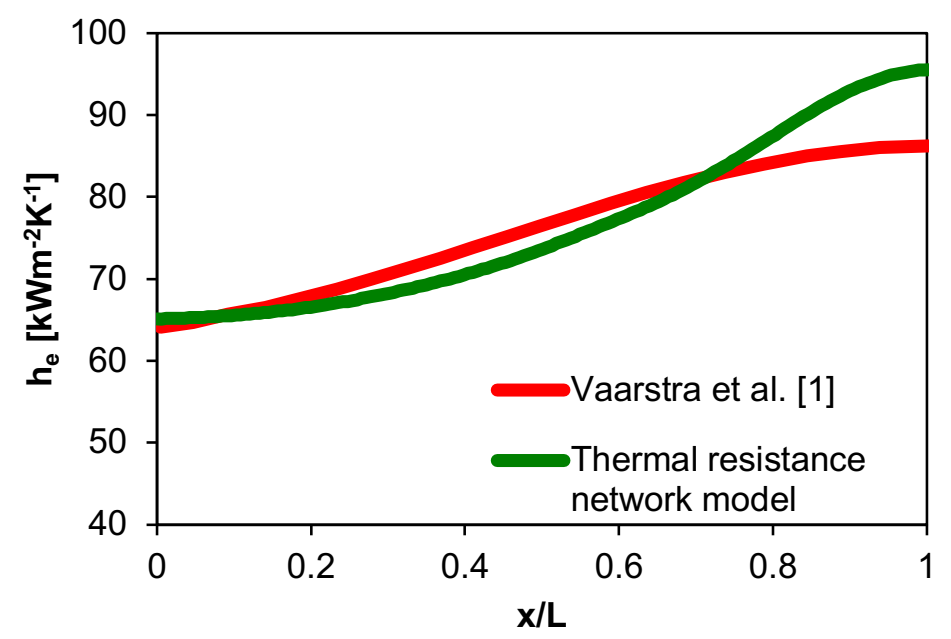

Figure S1. Comparison of effective heat transfer coefficient calculated with our thermal resistance network model against full numerical simulation results from Vaarstra et al. [1] for evaporation from a uniform micropillar array with $d=10 \mu \mathrm{m}, p=30 \mu \mathrm{m}$, and $h=25 \mu \mathrm{m}, q "=82 \mathrm{~W} / \mathrm{cm}^{2}$, and length $L=5 \mathrm{~mm}$. The resistance network model shows good agreement with the full numerical simulation results, validating the simplified model assumptions.

\section{SECTION II: Contact angle recovery during transitions between micropillar segments}

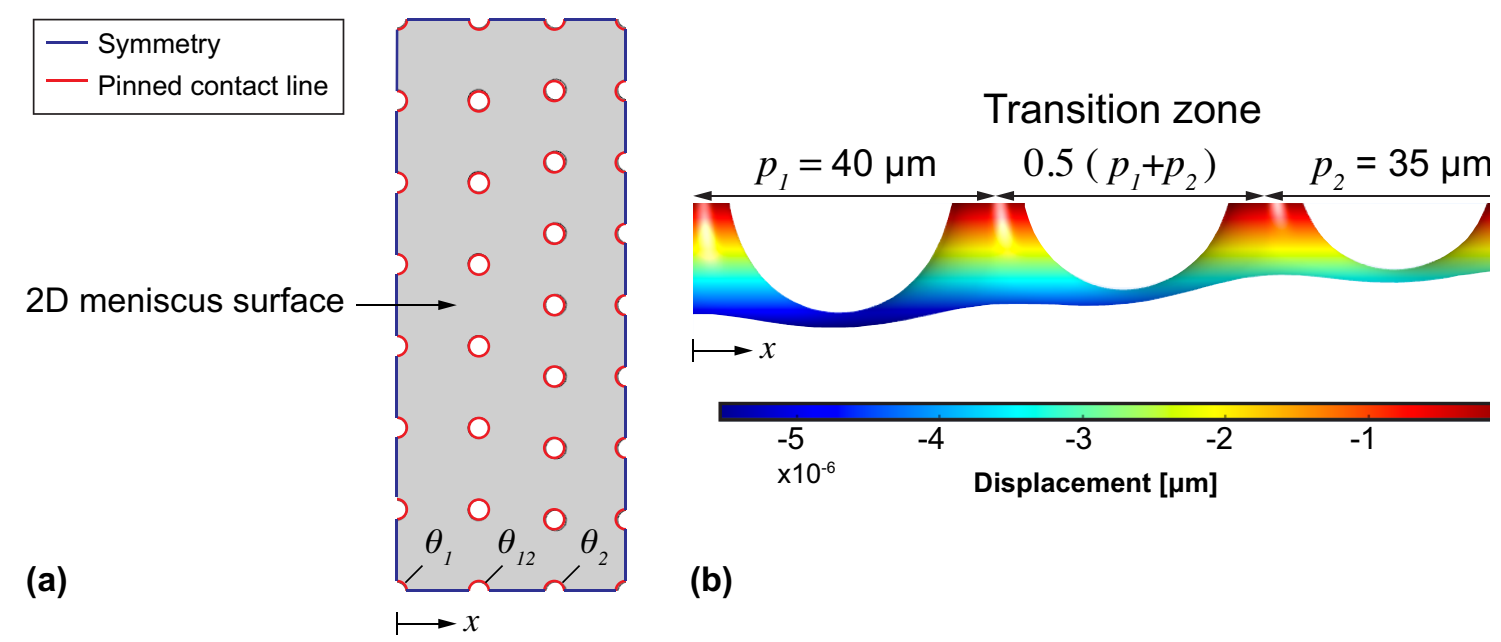

Figure S2. (a) Top view of example meniscus portion modeled in COMSOL to examine contact angle recovery during transitions between micropillars of different geometry. The red lines represent pinned contact line constraints, and the remaining boundaries are assigned symmetry conditions. (b) Side view of computed meniscus displacement for configuration shown in (a) subject to a vapor-liquid pressure difference of $-1 \mathrm{kPa}$. Note that the displacement contour has been scaled by a factor of 5 to aid in the visualization. 
We used a detailed force balance model in COMSOL to confirm the type of sharp contact angle recovery observed in Figure 3(b) of the main text. A portion of an example meniscus is modeled as a 2D surface as shown in Figure S2(a). The specific surface shown in Figure S2(a) is representative of a transition between pillars with $d_{l}=10 \mu \mathrm{m}, p_{1}=40 \mu \mathrm{m}$, to $d_{2}=10 \mu \mathrm{m}, p_{2}=35$ $\mu \mathrm{m}$. As seen in Figure S2(a), we evaluate the contact angles at three locations throughout the wick. $\theta_{1}$ is located in the region before the transition with $p_{1}=40 \mu \mathrm{m}, \theta_{12}$ is located in the transition zone, and $\theta_{2}$ is located in the region after the transition with $p_{2}=35 \mu \mathrm{m}$. Due to the geometric asymmetry surrounding the pillars in the transition zone, the contact angle is non-uniform around the periphery of the micropillar. We therefore evaluate the contact angles at each location along the segment of the meniscus profile that extends from the micropillar surface towards the center of each unit cell, or along a line situated at an angle of approximately $45^{\circ}$ from the $x$-direction. This gives an estimate of the lowest contact angle around the micropillar periphery. The same criteria is used for all contact angles calculated in the unit cell model, though minimal variation of the contact angle is expected around the peripheral of micropillars outside of the transition regions [2].

Pinned constraints are applied to the micropillar contact lines and symmetry conditions are applied to the remaining borders. The meniscus curvature and resulting contact angles are then calculated numerically by solving the Young-Laplace equation over the surface subject to an applied vapor-liquid pressure difference. Figure S2(b) shows the displacement output by the simulation along a cross section of the meniscus surface for an applied pressure difference of -1 $\mathrm{kPa}$. The sparser pillar array $\left(p_{1}=40 \mu \mathrm{m}\right)$ has a noticeably higher displacement that gradually reduces in the denser array $\left(p_{2}=35 \mu \mathrm{m}\right)$. This results in a contact angle that increases along the meniscus transition zone, where $\theta_{1}=36^{\circ}, \theta_{12}=42^{\circ}$, and $\theta_{2}=51^{\circ}$, confirming the validity of the type of contact angle recovery observed in Figure 3 of the main text.

\section{SECTION III: Analytical approximation for rapid Pareto front generation}

An analytical approximation to calculate the dryout heat flux is used for rapid generation of the Pareto front during the genetic algorithm optimization. To provide context, iterating for the dryout heat flux with the detailed unit cell model over a wicking length of $1 \mathrm{~cm}^{2}$ can require the solution of more than 1000 equations based on the range of micropillar pitches considered. On a standard computer with Intel Core 17 processors, this requires on average at least 10 minutes per solution. The genetic algorithm has a population of 200 individuals per generation and can require more than 100 generations before converging, leading to multiple days of computational time to complete the optimization with the full unit cell model. 
Therefore, we use a simplified model during the optimization procedure to calculate the dryout heat flux. The simplified model uses a Darcy's Law approximation to solve for the pressure drop along each $n$th wick segment with an average permeability value. The average permeability value, $K_{n}$, is extracted for each micropillar geometry from the tabulated CFD results for $U$ as a function of unit cell pressure drop as described in section 2.1 of the main text. Figure S3 shows an example pressure gradient versus average velocity curve from the CFD results for the micropillar wick geometry M5 ( $d=10 \mu \mathrm{m}, p=30 \mu \mathrm{m}$, and $h=50 \mu \mathrm{m})$ with a vapor-liquid pressure difference of $-1.1 \mathrm{kPa}$.

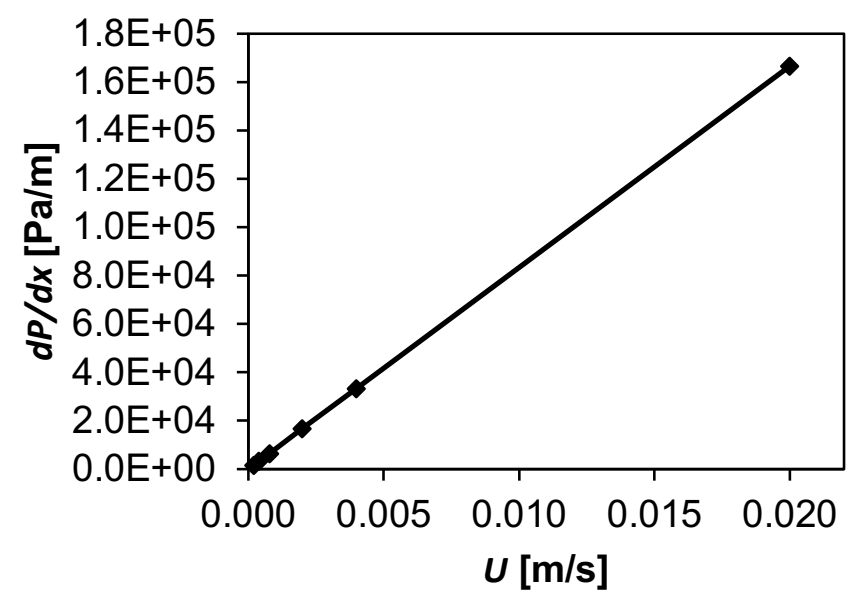

Figure S3. Plot of pressure gradient versus average velocity extracted from CFD simulations of flow through a micropillar unit cell for wick geometry M5 $(d=10 \mu \mathrm{m}, p=30 \mu \mathrm{m}$, and $h$ $=50 \mu \mathrm{m}$ ) with a vapor-liquid pressure difference of $-1.1 \mathrm{kPa}$. The permeability for this configuration is extracted through a linear fit with Darcy's Law.

The permeability for a given vapor-liquid pressure difference, or meniscus shape can be extracted from a linear fit based on Darcy's Law, where

$$
\frac{d P}{d x}=\frac{-\mu}{K} U
$$

and $\mu$ is the dynamic viscosity of the fluid. Note that since $U$ is the average fluid velocity evaluated at the center of the unit cell, it is equivalent to the superficial velocity used in Darcy's Law. The average permeability for a given wick geometry, $K_{n}$ is then found by taking the average of the individual permeabilities extracted for a series of vapor-liquid pressure differences ranging from a 0 (flat meniscus) to the maximum sustainable capillary pressure difference, $P_{c, \max }$ (dryout condition). We then apply Darcy's Law subject to an external mass flux to solve for the pressure drop within each wick segment using the average permeability values. 
As seen in Figure S4, each segment is assigned a local $x$ coordinate that ranges from 0 - $L_{n}$, or the length of the segment.

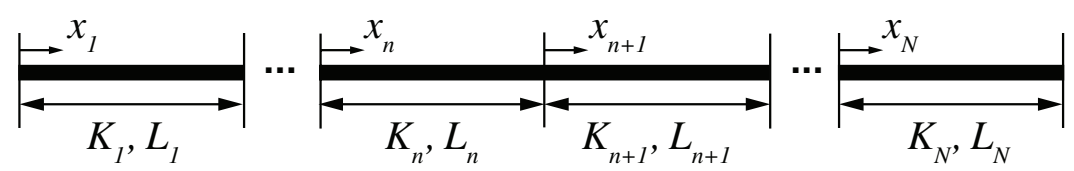

Figure S4. Schematic of modeling approach used in analytical approximation to predict the dryout heat flux in a segmented wick. Each segment has a local coordinate, $x_{\mathrm{n}}$, that varies from 0 to the length of each segment, $L_{n}$.

From a mass balance over the $n$th wick segment, we have

$$
\rho h w U_{0, n}=\frac{q_{n}^{\prime \prime} w x_{n}}{h_{f g}}+\rho h w U\left(x_{n}\right)
$$

where $w$ is the width of the wick cross sectional to the flow direction, $q_{n}$ " is the heat flux applied to the base of the particular wick segment, and $U_{0, n}$ is the initial flow velocity entering the segment. The value of $q_{n}$ " that will lead to dryout within the wick is an unknown at this point.

Substituting Eqn. S1 into Eqn. S2 and integrating with respect to $x$ leads to the following equation for the relative pressure drop within each wick segment as a function of the local $x$ coordinate $x_{n}$, where

$$
\left.P\left(x_{n}\right)=\frac{\mu}{\rho h K_{n}}\left(\frac{q_{n}^{\prime \prime} x_{n}^{2}}{2 h_{f g}}-C_{1, n} x_{n}+C_{2, n}\right)\right)
$$

$C_{1}$ and $C_{2}$ are constants of integration and can be solved for by applying the appropriate boundary conditions at the end of each segment. $C_{l}$ represents the mass flow entering the start of the wick from the previous wick segment and can be calculated by evaluating the derivative of Eqn. S3 at $x_{n}$ $=0$ and $x_{n-1}=L_{n-1}$, then using Eqn. S1 to enforce mass flow continuity. This gives the following relation linking the different segments, where

$$
C_{1, n} K_{n}=\left(\frac{q_{n-1}^{\prime \prime} \mu x_{n-1}{ }^{2}}{h \rho h_{f g} K_{n-1}} L_{n-1}+C_{1, n-1}\right) K_{n-1}
$$

Enforcing pressure continuity across the segments leads to the second relation where

$$
C_{2, n}=P\left(x_{n-1}=L_{n-1}\right)
$$


Finally, we set $P\left(x_{n}=L_{n}\right)=P_{c, \max }$ to enforce the dryout criterion in the given segment of interest and assume that the relative pressure at the start of the entire wick is zero, or $P\left(x_{1}=0\right)=0$. The dryout heat flux for a given heating configuration can then be solved from the analytical equations with these additional boundary conditions.

We note that this analytical method is a very good approximation to the full unit cell model when the effect of local meniscus shape on the wick permeability is relatively small. For the micropillar geometries considered in this study, the error in predicted dryout heat flux for the analytical model compared to the full unit cell model is typically less than $2 \%$. For relatively sparse, short pillar arrays, however, the greater impact of meniscus shape on permeability may lead to less accurate predictions with the analytical model.

\section{SECTION IV: Genetic algorithm solver and stopping criteria}

We use the genetic algorithm toolbox in MATLAB to solve the multi-objective optimization problem of minimizing the thermal resistance and maximizing the dryout heat flux. The toolbox uses a variant of the NSGA-II algorithm to create a natural selection approach to find the set of individuals that optimizes the objective function. An initial population of individuals is first generated at random. Each individual is then ranked based on a fitness function. Depending on the rank, parents are selected that may be passed directly onwards to the next generation as an elite, or have its parameters mutated or swapped with another individual (crossover) to form a new child [3]. The algorithm continues in this cycle until the average change in the fitness function between generations is less than or equal to a predefined tolerance.

Since the solver is built to perform a multi-objective minimization, we use the negative of the computed dryout heat flux as the first component of the fitness function. The second component is the thermal resistance across the wick, computed based on the meniscus distribution output from the fluid flow model as outlined in section 2.2. The total number of optimization variables is the number of discrete wick geometry segments implemented in the final wick. As mentioned in section 3.2 , the $n$ variables are constrained to a value $\alpha_{\mathrm{n}}$ ranging from $0-1$. To prevent calculation errors arising from small values of $\alpha_{\mathrm{n}}$ that may lead to segment lengths less than or comparable to a single unit cell size, we add the additional criteria that at minimum, each segment must consist of at least 4 unit cells.

The initial population for the genetic algorithm is specified as 200 individuals. The crossover fraction is set at 0.8 , and the default mutation and crossover functions for problems with linear constraints are chosen. A tournament selection function is used to determine parents for the next generation. To ensure the accuracy of the solutions and convergence on global optima as opposed to local optima, multiple runs were performed for each optimization configuration. As 
seen in Figure S5, the generated Pareto front for the case of a $1 \times 1 \mathrm{~cm}^{2}$ wick area, $5 \mathrm{~mm}$ heated length, and micropillar geometries M1-8 remains consistent across three different runs.



Figure S5. Pareto front results for a $1 \times 1 \mathrm{~cm}^{2}$ wick area, $5 \mathrm{~mm}$ heated length, and all micropillar geometries M1-8 across three different runs. Since the initial population is chosen at random, multiple runs with overlapping results confirm that the generated Pareto fronts consist of global optima as opposed to local optima.

\section{REFERENCES}

[1] G. Vaartstra, Z. Lu, E.N. Wang, Simultaneous prediction of dryout heat flux and local temperature for thin film evaporation in micropillar wicks, Int. J. Heat Mass Transf. 136 (2019) 170-177. doi:10.1016/j.ijheatmasstransfer.2019.02.074.

[2] Y. Zhu, D.S. Antao, Z. Lu, S. Somasundaram, T. Zhang, E.N. Wang, Prediction and Characterization of Dry-out Heat Flux in Micropillar Wick Structures, Langmuir. 32 (2016) 1920-1927. doi:10.1021/acs.langmuir.5b04502.

[3] S. Huband, P. Hingston, L. Barone, L. While, A review of multiobjective test problems and a scalable test problem toolkit, IEEE Trans. Evol. Comput. 10 (2006) 477-506. doi:10.1109/TEVC.2005.861417. 